\title{
Mechanical Properties and Fracture Toughness of Epoxy Resin Improved by Low-viscosity Hyperbranched Epoxy
}

\author{
Zhongwei ZHANG ${ }^{1, a}$, Xiaolong WANG ${ }^{1, b^{*}}$, Xiaoguang $\mathrm{HU}^{2, c}$, Ting XU ${ }^{1, d}$, \\ Yanyan LIN $^{1, e}$, and Yefa TAN ${ }^{1, f}$ \\ ${ }^{1}$ College of Field Engineering, PLA University of Science and Technology, Nanjing 210007, China \\ ${ }^{2}$ Military Representative Office of Engineering Corps in Wuhan Area, Wuhan 430073, China

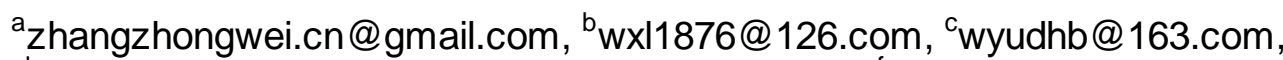 \\ dxuting3521064@126.com, ${ }^{\mathrm{e}} 648890232 @ q q . c o m,{ }^{\mathrm{f}}$ tanyefa7651@163.com
}

Keywords: mechanical property, fracture toughness, epoxy, hyperbranched, low-viscosity

Abstract. Epoxy resins modified with low-viscosity hyperbranched epoxy were prepared. The tensile strength, flexural strength, impact strength, fracture toughness, glass transition temperature (Tg) of the cured samples were investigated. The results show that the addition of hyperbranched epoxy improves the overall mechanical and fracture performance because of the unique three-dimensional macromolecule structure. With the content of hyperbranched epoxy increases, the tensile strength, flexural strength, impact strength and fracture toughness all increases first and then decreases. This variation trend can be attributed to the reverse effect of crosslinking density and the rigidity of molecule chain of the hyperbranched epoxy. The Tg of the cured samples decreases in the whole range studied. The decrease of viscosity may be contributive to developing an easier fabrication process of the epoxy based materials.

\section{Introduction}

Epoxy resins (EPs) show several advantages such as high modulus, high strength, outstanding chemical resistance and excellent adhesion to numerous substrates, thus they are widely used in many engineering applications [1-3]. However, due to the tight crosslinking structure, EPs also present poor crack resistance and brittle fracture behavior, which usually limits their broader applications. Therefore, toughening and reinforcing the EPs are considered one of the key issues in epoxy industry. Up to now, EPs have been toughened and reinforced by rubber [4], rigid micron particles [5] or nanofillers [6]. Most of these means toughen or reinforce the EPs at the expense of other properties. For example, liquid rubber can improve the fracture toughness ofEPs effectively, but the strength and modulus of EPs will decrease sharply. And several kinds of nanofillers can simultaneously improve the mechanical performance and fracture toughness of the EPs, however, an undesirable rise in viscosity usually hampers the easy processing property of them.

Hyperbranched polymers (HBPs) are a category of macromolecules with three-dimensional structures. They show lower viscosity than those of the linear polymers at the same molecular weight owing to the unique structure. With many active groups on the molecule surfaces, HBPs have great potential in improving the fracture toughness of EPs [7]. At present, HBPs are researched in different applications, and already commercialized as reactive components in coatings and resin formulations.

In this study, a hyperbranched epoxy was used to toughen and reinforce the EPs. The content of hyperbranched epoxy was $3 \mathrm{phr}-15 \mathrm{phr}$. Scanning electron microscopy (SEM) observation, differential scanning calorimetry (DSC) analysis and mechanical testing were used to investigate the effect of hyperbranched epoxy content on the microstructure, thermal performance, mechanical properties and fracture toughness of the epoxy resin. The main aim of this research is to improve the overall performance of the EPs without sacrifice of other key mechanical properties, thus providing technological guidance for the epoxy based materials in engineering applications. 


\section{Experimental}

Materials. The hyperbranched epoxy was provided by Wuhan Hyperbranched Resins Science \& Technology, China, and its main parameters are listed in Table 1. The epoxy matrix utilized was a bisphenol A epoxy resin (WSR618) with an epoxy equivalent of 185-192g/eq from Bluestar Wuxi Petrochemical Co., Ltd, China. The curing agent was FS-2B from Chuzhou Hui-Sheng Electronic Materials Co., Ltd, China.

Table 1 Properties of the hyperbranched epoxy.

\begin{tabular}{cccc}
\hline $\begin{array}{c}\text { Molecular weight } \\
{[\mathrm{g} / \mathrm{mol}]}\end{array}$ & $\begin{array}{c}\text { Epoxy equivalent } \\
\text { weight }[\mathrm{g} / \mathrm{mol}]\end{array}$ & $\begin{array}{c}\text { Viscosity } \\
{[\mathrm{cp}]}\end{array}$ & $\begin{array}{c}\text { Molecular weight } \\
\text { distribution }\left[\mathrm{M}_{\mathrm{w}} / \mathrm{M}_{\mathrm{n}}\right]\end{array}$ \\
\hline 3400 & 400.0 & 700 & 2.06 \\
\hline
\end{tabular}

Sample preparation. Firstly, the hyperbranched epoxy was mixed with the epoxy resin according to the content needed in this study, so that the epoxy blend was obtained. Secondly, the epoxy blend was mixed with the curing agent. Finally, the samples were cured in a stainless steel mold according to the manufacturer's recommended curing parameters.

Characterization. The tensile strength and flexural strength of the cured samples were tested on a SANS CMT5105 universal testing machine at the temperature of $23^{\circ} \mathrm{C}$, according to the standard of ASTM D638-2010 and ASTM D790-03, respectively. The impact strength was studied on a XJ-6608C impact instrument in terms of un-notched sample at $25{ }^{\circ} \mathrm{C}$. The geometries of the impact samples are $80 \mathrm{~mm} \times 10 \mathrm{~mm} \times 4 \mathrm{~mm}$ according to the ASTM D256 standard.

The fracture toughness of the composites was determined by way of the single edge notched bend (SENB) specimens in accordance with ASTM D5045-99. The rectangular coupons were cured in a steel mould with dimensions of $60 \mathrm{~mm} \times 10 \mathrm{~mm} \times 5 \mathrm{~mm}$. Fracture properties were characterized in terms of the fracture toughness $\left(\mathrm{K}_{\mathrm{IC}}\right)$, which was listed as the following equations(1) [8]. The Possion ratios of the materials studied were 0.35 [9].

$$
K_{I C}=Y \frac{6 P_{f} S}{4 t w^{2}} \sqrt{a}
$$

The glass transition temperature ( $\mathrm{Tg}$ ) of the composites was measured by a Differential Scanning Calorimetry (DSC, Mettler-Toledo DSC823e, Switzerland). All the measurements were carried on under nitrogen atmosphere with a sample mass of $15 \mathrm{mg}$. The samples were tested with a temperature range of $50 \sim 300^{\circ} \mathrm{C}$ at a scanning rate of $10^{\circ} \mathrm{C} / \mathrm{min}$. The viscosity of the blend was tested on a Rheogeniometer (HAAKE MARS, Thermo Fisher Scientific) according the ASTM D1084-2008 standard. The scanning electron microscope (SEM, Hitachi S4800, Japan) was used to examine the morphologies of the fracture surfaces.

\section{Results and discussion}

Tensile and flexural strength. Effect of hyperbranched epoxy content on tensile strength of the cured samples is presented in Fig. 1. It can be observed that the tensile strength increases first and then decreases in the whole range. And it achieves the maximum value (71.1MPa) at the hyperbranched epoxy content of 9phr, increasing by $19.3 \%$ than that of the neat EP. The tensile strength of a cured sample is usually affected by the crosslinking density and the rigidity of molecule chain. In general, with the increasing content of hyperbranched epoxy, the crosslinking density of the cured system decreases. The hyperbranched epoxy used in this study has many rigid benzene rings in the molecule chain [10]. Therefore, with the increasing content of hyperbranched epoxy, the rigidity of molecule chain shows positive effect while the crosslinking density has negative effect on the tensile strength. Due to the reverse effect on tensile strength of the cured system, the variation trend above of the tensile strength was formed.

Fig. 2 shows the effect of the hyperbranched epoxy content on tensile strength of the cured system. As seen in the figure, the flexural strength presents similar variation trend as the tensile strength. Different with the tensile strength, the flexural strength achieves the maximum value (112.4MPa) at the hyperbranched epoxy 
content of $12 \mathrm{phr}$, increasing by $28.0 \%$ than that of the neat EP. When the hyperbranched epoxy content increases from $12 \mathrm{phr}$ to $15 \mathrm{phr}$, the flexural strength shows a sharp decrease. This phenomenon may also be attributed to the reverse effect of crosslinking density and the rigidity of molecule chain on flexural strength of the cured system.

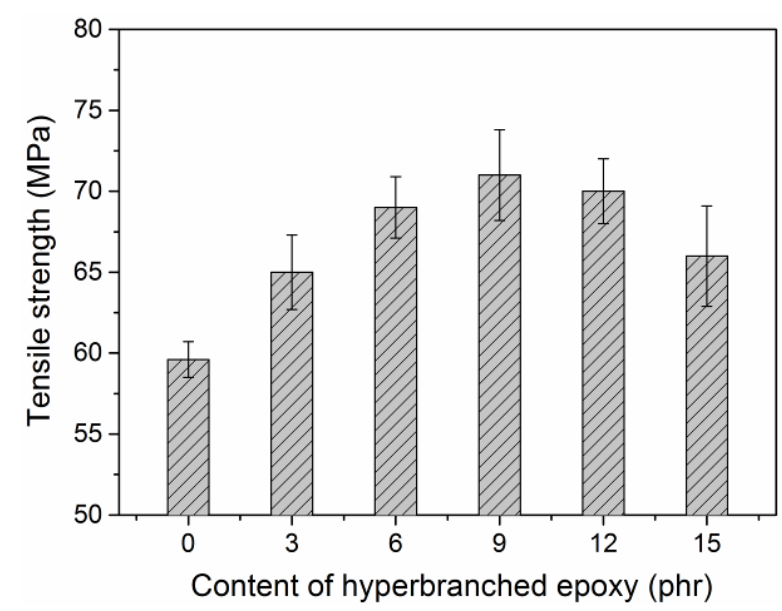

Fig.1 Effect of hyperbranched epoxy content on tensile strength of the epoxy resin.

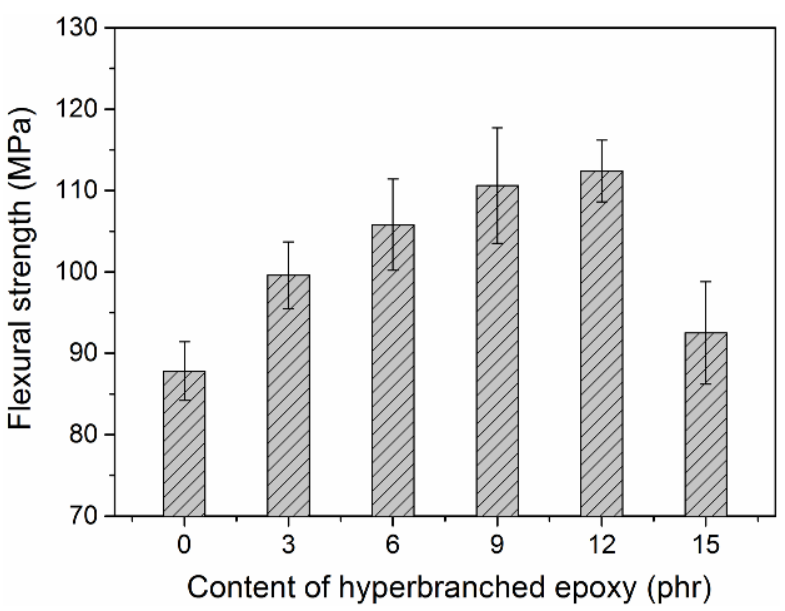

Fig.2 Effect of hyperbranched epoxy content on flexural strength of the epoxy resin

Impact strength and fracture toughness. Effect of hyperbranched epoxy content on tensile strength of the cured system is shown in Fig.3. It can be seen that all the filled samples show enhancement on impact strength. When the hyperbranched epoxy content increases to $9 \mathrm{phr}$, the impact reaches its maximum value of $36.4 \mathrm{KJ} / \mathrm{m}^{2}$, increasing by $195.9 \%$ than that of the neat EP. Meanwhile, the fracture toughness increases first and then decreases in the whole range (Fig.4). It achieves the maximum value of $1.35 \mathrm{MPa} \cdot \mathrm{m}^{1 / 2}$, increasing by $104.5 \%$ than that of the neat EP. The impact strength and fracture toughness reflect the crack resistance ability under dynamic and quasi-static conditions, respectively. Combined with the results obtained in Fig. 1 and Fig.2, the overall improvement of tensile strength, flexural strength, impact strength and fracture toughness indicates that the addition of hyperbranched epoxy contributes to improving not only the mechanical strength but also the fracture performance of the neat EP.

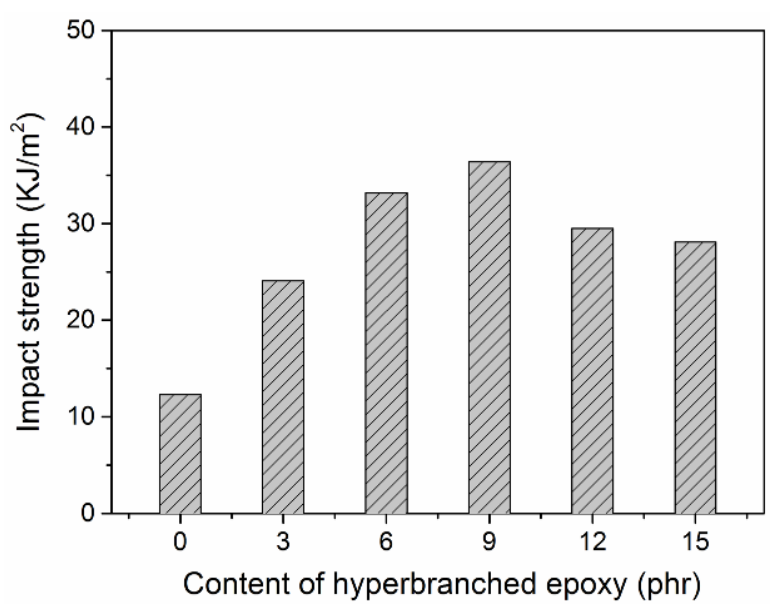

Fig.3 Effect of hyperbranched epoxy content on impact strength of the epoxy resin.

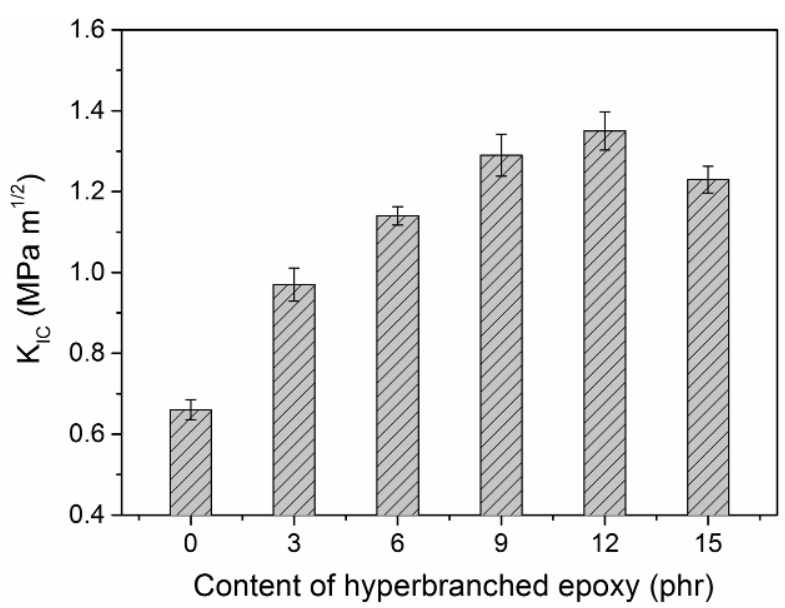

Fig.4 Effect of hyperbranched epoxy content on fracture strength of the epoxy resin.

Glass transition temperature and viscosity test. Fig.5 shows the effect of hyperbranched epoxy content on $\mathrm{Tg}$ of the cured system. As seen in the figure, $\mathrm{Tg}$ decreases from $128.4{ }^{\circ} \mathrm{C}$ to $92.3{ }^{\circ} \mathrm{C}$ with the hyperbranched epoxy content increases from $3 \mathrm{phr}$ to $15 \mathrm{phr}$, and this decline trend gradually slows down. When the hyperbranched epoxy content further increases to $15 \mathrm{phr}$, $\mathrm{Tg}$ of the cured system remains almost unchanged. The molecular weight of the hyperbranched epoxy used in this work was much greater than that 
of the epoxy resin, thus the increasing content of hyperbranched epoxy will reduce the crosslinking degree of the cured system. And the decrease of crosslinking degree usually means a lower Tg. Similar reports also exist in the published literature [10]. The viscosity of the epoxy blend before curing usually affects the difficulty degree of processing. Therefore, the viscosity is also considered a key property of the epoxy blend. Fig.6 shows the viscosity testing results of the epoxy blend filled with different content of hyperbranched epoxy. As the hyperbranched epoxy content increases from $3 \mathrm{phr}$ to $15 \mathrm{phr}$, the viscosity decreases from $12.1 \mathrm{~Pa} \cdot \mathrm{s}$ to $10.1 \mathrm{~Pa} \cdot \mathrm{s}$, decreasing by $16.5 \%$ than that of the neat EP. Hyperbranched polymers are macromolecules with three dimensional structures, thus their viscosity is lower than that of linear molecules in the same molecular weight because of their special structure. Different with the addition of micron or nano sized fillers into an epoxy blend, the addition of hyperbranched epoxy decreases the viscosity of the epoxy blend instead of increasing it, which contributes to make it easier to fabricate the epoxy based materials.

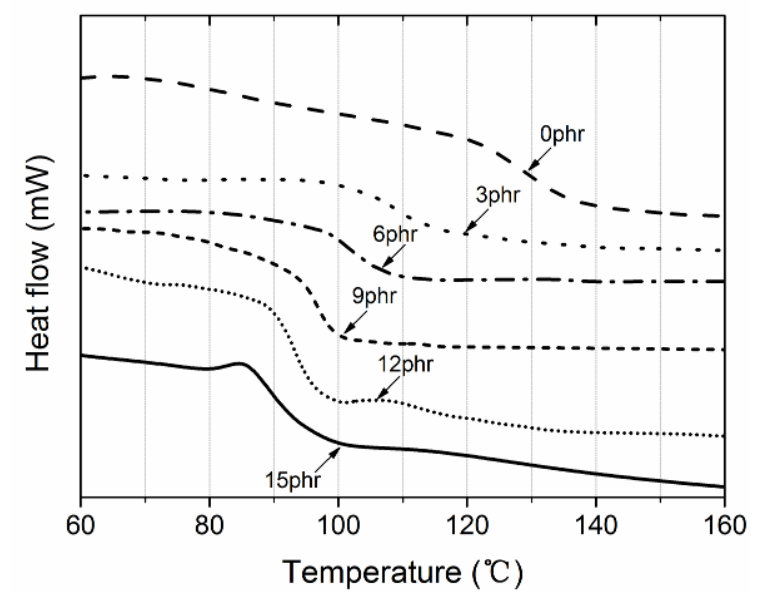

Fig.5 Effect of hyperbranched epoxy content on Tg of the epoxy resin.

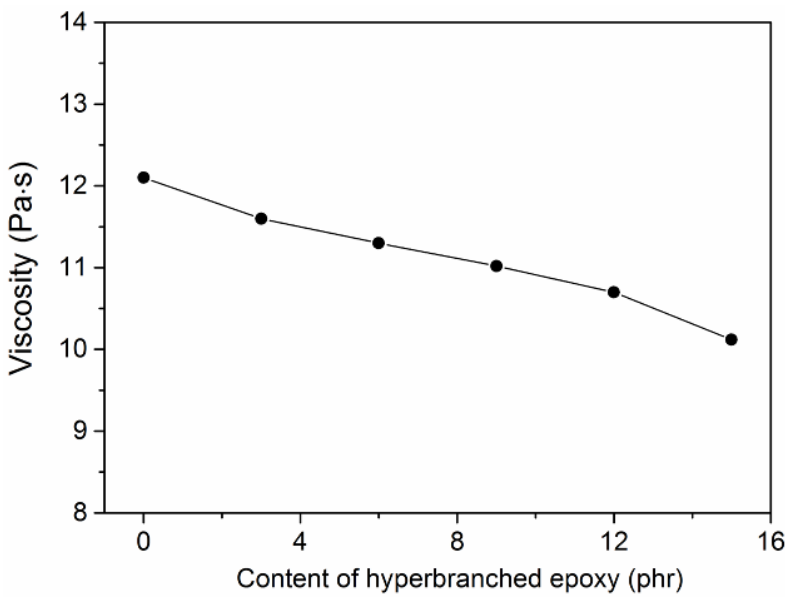

Fig.6 Effect of hyperbranched epoxy content on viscosity of the epoxy system.

Improvement mechanism. Fig.7 indicates the SEM micrographs of the fracture surfaces filled with different content of hyperbranched epoxy. As seen in the figure, the fracture surface of neat EP is very smooth (Fig.7a) because of the brittleness. This feature indicates a brittle and unstable crack propagation manner, which result from the low fracture toughness and fracture energy. After adding 3phr hyperbranched epoxy into the epoxy system, the fracture surface of the cured sample (Fig.7b) shows some crack propagation lines along the fracture direction. This phenomenon implies an improvement of the fracture toughness of the materials. When the hyperbranched epoxy further increases to $9 \mathrm{phr}$, the fracture surface is rougher with some filamentous features debonding away (Fig.7c). However, no phase separation was observed on the fracture surface. This toughening and reinforcing mechanisms can be described by in situ homogeneous mechanism [11]. From the above results, it can be found that with the addition of hyperbranched epoxy, the overall properties such as tensile strength, flexural strength, impact strength and fracture toughness are all improved. Moreover, the decrease of viscosity may be contributive to developing an easier fabrication process of the epoxy based materials.
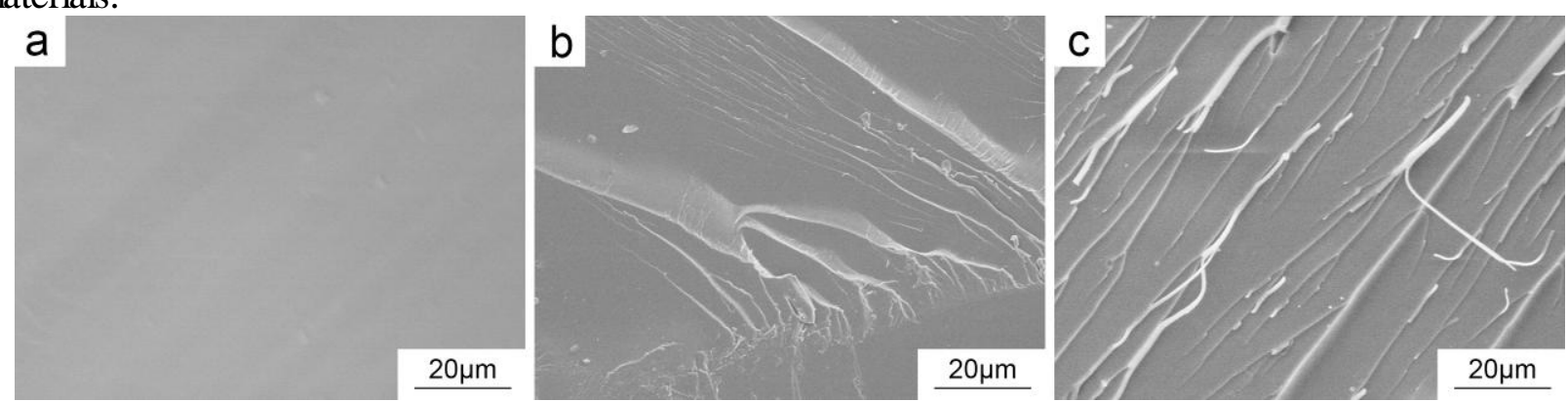

Fig.7 SEM micrographs of the fracture surface of hyperbranched epoxy filled system: (a) neat EP, (b) with 3 phr hyperbranched epoxy, (c) with 9phr hyperbranched epoxy. 


\section{Conclusions}

1) Epoxy resins modified with low-viscosity hyperbranched epoxy were prepared. The hyperbranched epoxy improves the overall mechanical and fracture performance because of the unique three-dimensional macromolecule structure.

2) The addition of hyperbranched epoxy increases the tensile strength, flexural strength, impact strength and fracture toughness of the cured samples, while decreases the Tg and viscosity of the materials.

3) With the content of hyperbranched epoxy increases, the tensile strength, flexural strength, impact strength and fracture toughness all increases first and then decreases. This variation trend can be attributed to the reverse effect of crosslinking density and the rigidity of molecule chain of the hyperbranched epoxy.

\section{References}

[1] H. Jin, G. M. Miller, N. R. Sottos, S. R. White, Fracture and fatigue response of a self-healing epoxy adhesive, Polymer 52 (2011) 1628-1634.

[2] K. Jung, J. Y. Bae, S. J. Park, S. Yoo, B. S. Bae, High performance organic-inorganic hybrid barrier coating for encapsulation of OLEDs, J. Mater. Chem. 21 (2011) 1977-1983.

[3] T. H. Ho, C. S. Wang, Modification of epoxy resin with siloxane containing phenol aralkyl epoxy resin for electronic encapsulation application, Eur. Polym. J. 37 (2001) 267-274.

[4] R. Bagheri, B. T. Marouf, R. A. Pearson, Rubber-toughened epoxies: a critical review, Polym. Rev. 49 (2009) 201-225.

[5] A. J. Kinloch, J. H. Lee, A. C. Taylor, S. Sprenger, C. Eger, D. Egan, Toughening structural adhesives via nano- and micro-phase inclusions, J. Adhesion 79 (2003) 867-873.

[6] S. Rana, R. Alagirusamy, M. Joshi, A review on carbon epoxy nanocomposites, J. Reinf. Plast. Comp. 28 (2008) 461-487.

[7] D. Yan, C. Gao, H. Frey, Hyperbranched polymers: synthesis, properties, and applications, John Wiley \& Sons, Singapore, 2011.

[8] ASTM standard test methods for planestrain fracture toughness and strain energy release rate of plastic materials, D5045-99, 100 Barr Harbor Drive, PO Box C700, West Conshohocken, PA 19428-2959, United States, 1999.

[9] A.J. Kinloch, Adhension and adhensives science and technology, Chapman\&Hall, London, 1987.

[10] D. Zhang, D. Jia, Synthesis of novel low-viscosity liquid epoxidized aromatic hyperbranched polymers, Eur. Polym. J. 42(2006) 711-714.

[11] L. Boogh, B. Pettersson, J. E. Manson, Dendritic hyperbranched polymers as tougheners for epoxy resins, Polymer (1999) 2249-2261. 